\title{
Effective Theories and Modifications of Gravity
}

\author{
C.P. BURGESS \\ Department of Physics \& Astronomy, McMaster University, \\ 1280 Main St. W, Hamilton, Ontario, Canada, L8S $4 M 1$ \\ and \\ Perimeter Institute for Theoretical Physics, \\ 31 Caroline St. N, Waterloo, Ontario, Canada, N2L 2 Y5.
}

\begin{abstract}
We live at a time of contradictory messages about how successfully we understand gravity. General Relativity seems to work very well in the Earth's immediate neighborhood, but arguments abound that it needs modification at very small and/or very large distances. This essay tries to put this discussion into the broader context of similar situations in other areas of physics, and summarizes some of the lessons which our good understanding of gravity in the solar system has for proponents for its modification over very long and very short distances. The main message is that effective theories, in the technical sense of 'effective', provide the natural language for testing proposals, and so are also effective in the colloquial sense.
\end{abstract}

\subsection{Introduction}

Einstein's recognition early last century that gravity can be interpreted as the curvature of space and time represented an enormous step forward in the way we think about fundamental physics. Besides its obvious impact for understanding gravity over astrophysical distances complete with resolutions of earlier puzzles (like the detailed properties of Mercury's orbit) and novel predictions for new phenomena (like the bending of light and the slowing of clocks by gravitational fields) - its implications for other branches of physics have been equally profound.

These implications include many ideas we nowadays take for granted. One such is the universal association of fundamental degrees of freedom with fields (first identified for electromagnetism, but then cemented with its extension to gravity, together with the universal relativistic rejection 
of action at a distance). Another is the recognition of the power of symmetries in the framing of physical law, and the ubiquity in particular of gauge symmetries in their description (again reinforcing the earlier discovery in electromagnetism). A third is the systematization of the belief that the physical content Nature's laws should be independent of the variables used in their description, and the consequent widespread penetration of geometrical methods throughout physics.

But the study of General Relativity (GR) and other interactions (like electromagnetism, and its later-discovered relatives: the weak and strong forces) have since drifted apart. Like ex-lovers who remain friends, for most of the last century practitioners in either area have known little of the nitty gritty of each other's day-to-day struggles, even as they read approvingly of their occasional triumphs in the popular press.

Over the years the study of both gravity and the other interactions has matured into precision science, with many impressive theoretical developments and observational tests. For gravity this includes remarkably accurate accounts of motion within the solar system, to the point that GR - through its use within the global positioning system (GPS) - is now an indispensable tool for engineers [Will 2001]. For the other interactions the successes include the development and testing of the Standard Model (SM), a unified framework for all known non-gravitational physics, building on the earlier successes of Quantum Electrodynamics (QED).

There is nevertheless a mounting chorus of calls for modifying General Relativity, both at very short and very long distances. These arise due to perceived failures of the theory when applied over distances much different from those over which it is well-tested. The failures at short distances are conceptual, to do with combining gravity with quantum effects. Those at long distances are instead observational, and usually arise as ways to avoid the necessity for introducing the dark matter or dark energy that seem to be required when General Relativity is applied to describe the properties of the universe as a whole.

The remainder of this chapter argues that when searching for replacements for GR over short and long distances there is much to be learned from other branches of physics, where similar searches have revealed general constraints on how physics at different scales can relate to one another. The hard-won lessons learned there also have implications for gravitational physics, and this recognition is beginning to re-establish the connections between the gravitational and non-gravitational research communities. 
In a nutshell, the lessons distilled from other areas of physics make it likely that it is much more difficult to modify gravity over very long distances than over very tiny ones. This is because very broad principles (like unitarity and stability) strongly restrict what is possible. The difficulty of modifying gravity over long distances is a very useful (but often neglected) clue when interpreting cosmological data, because it strongly constrains the theoretical options that are available. We ignore such clues at our peril.

This chapter is also meant to be colloquial rather than authoritative, and so citations are not thorough. My apologies to those whose work is not properly cited.

\subsection{Modifying Gravity over Short Distances}

The demand to replace General Relativity at short distances arises because quantum mechanics should make it impossible to have a spacetime description of geometry for arbitrarily small scales. For example, an accurate measurement of a geometry's curvature, $R$, requires positions to be measured with an accuracy, $\delta$, smaller than the radius of curvature:

$$
\delta^{2}<1 / R .
$$

But for position measurements with resolution, $\delta$, the uncertainty principle requires a momentum uncertainty, $p \simeq \hbar / \delta$, which implies an associated energy uncertainty, $E \simeq p c \simeq \hbar c / \delta$, or equivalently a mass $M \simeq E / c^{2} \simeq \hbar / \delta c$. But the curvature associated with having this much energy within a distance of order $\delta$ is then $R \simeq G M / \delta^{3} c^{2} \simeq G \hbar / \delta^{4} c^{3}=$ $\ell_{p}^{2} / \delta^{4}$, where $\ell_{p}$ defines the Planck length, $\ell_{p}^{2}=G \hbar / c^{3}$, and $G$ is Newton's constant. Requiring eq. (1.1), then shows that there is a lower bound on the resolution with which spacetime can be measured:

$$
\delta>\ell_{p} \simeq \sqrt{\frac{G \hbar}{c^{3}}} \simeq 1.6 \times 10^{-35} \mathrm{~m} .
$$

Although this is an extremely short distance (present experiments only reach down to about $10^{-19} \mathrm{~m}$ ), it is also only a lower bound. Depending on how gravity really works over short distances, quantum gravity effects could arise at much longer scales.

Notice how crucial it is to this argument that the interaction strength, $G$, has dimensions of length (in fundamental units, for which $\hbar=c=1$ ). Imagine performing a similar estimate for an electrostatic field. The 
Coulomb interaction energy between two electrons separated by a distance $\delta$ is $E_{c} \simeq e^{2} / \delta$, where $q=-e$ denotes the electron's electric charge. But the energy required by the uncertainty principle to localize electrons this close to one another is $E \simeq \hbar c / \delta$, so the condition that this be smaller than $E_{c}$ is

$$
\alpha=\frac{e^{2}}{4 \pi \hbar c}<1
$$

where the fine-structure constant, $\alpha \simeq 1 / 137$, is dimensionless. This condition doesn't depend on $\delta$ because the relative strength of quantum fluctuations to electrostatic interactions does not change with distance.

\subsubsection{Gravity and renormalizability}

The observation that quantum fluctuations do not get worse at shorter distances in electrodynamics $\$$ but do for gravity can be more technically expressed as the statement that QED is a renormalizable quantum field theory (QFT) while GR is not. In QFT small-distance quantum fluctuations appear (within perturbation theory) as divergences at small distances (or high momenta) when summing over all possible quantum intermediate states.

For instance, given a Hamiltonian, $H=H_{0}+H_{\text {int }}$, the second-order shift in the energy of a state $|n\rangle$ is

$$
\delta_{2} E_{n}=\sum_{m} \frac{\left|\left\langle n\left|H_{\mathrm{int}}\right| m\right\rangle\right|^{2}}{E_{m}-E_{n}} \simeq \int \frac{\mathrm{d}^{3} \mathbf{p}}{(2 \pi)^{3}} \frac{\left|\left\langle n\left|H_{\mathrm{int}}\right| \mathbf{p}\right\rangle\right|^{2}}{E(\mathbf{p})-E_{n}}+\cdots,
$$

where the approximate equality focusses on the sum over a basis of free single-particle states having energies $E(\mathbf{p})=\sqrt{\mathbf{p}^{2}+m^{2}}$ when performing the sum over $|m\rangle$. Because the combination $\left|\left\langle n\left|H_{\text {int }}\right| \mathbf{p}\right\rangle\right|^{2} /\left[E(\mathbf{p})-E_{n}\right]$ typically falls with large $p=|\mathbf{p}|$ like $1 / p^{3}$ or slower, the integration over the momentum of the intermediate state diverges in the ultraviolet (UV), $p \rightarrow \infty$, limit. (Relativistic calculations organize these sums differently to preserve manifest Lorentz invariance at each step, but the upshot is the same.)

Renormalizability means that these divergences can all be absorbed into the unknown parameters of the theory - like the electron's charge and mass, for instance — whose values must in any case be inferred

$\dagger$ There is a sense in which quantum effects in QED do get worse at smaller distances, because the theory is not asymptotically free. But this problem only arises logarithmically in $\delta$, and so is much less severe than the power-law competition found above for gravity. 
by comparison with experiments. As the above estimates suggest, the hallmark of a nonrenormalizable theory is the appearance of couplings (like Newton's constant) having dimensions of length to a positive power (in fundamental units). Couplings like this ruin perturbative renormalizability because the more powers of them that appear in a result, the more divergent that result typically is.

For instance, a contribution that arises at $n$th order in Newton's constant usually depends on $G$ through the dimensionless combination $\left(G \Lambda^{2}\right)^{n} \propto\left(\ell_{p} / \delta\right)^{2 n}$, where $\Lambda \propto 1 / \delta$ is the UV cutoff in momentum space (equivalently, $\delta$ is the small-distance cutoff in position space). By contrast, having more powers of dimensionless couplings, or those having dimensions of inverse powers of length, do not worsen UV divergences. Ever-worsening divergences ruin the arguments that show for renormalizable theories that all calculations are finite once a basic set of couplings are appropriately redefined. Removal of divergences can be accomplished, but only by introducing an infinite number of coupling parameters to be renormalized.

Lack of renormalizability was for a long time regarded as a fundamental obstacle to performing any quantum calculations within gravity. After all, if every calculation is associated with a new parameter that absorbs the new divergences, whose value must be inferred experimentally, then there are as many parameters as observables and no predictions are possible. If this were really true, it would mean that any classical prediction of GR would come with incalculable theoretical errors due to the uncontrolled size of the quantum corrections. And the presence of such errors would render meaningless any detailed comparisons between classical predictions and observations, potentially ruining GR's observational successes. How can meaningful calculations be made?

\subsubsection{Effective Field Theories}

As it happens, tools for making meaningful quantum calculations using non-renormalizable theories exist, having been developed for situations where quantum effects are more important than they usually are for gravity [Weinberg 1979, Gasser 1984].

The key to understanding how to work with non-renormalizable theories is to recognize that they can arise as approximations to more fundamental, renormalizable physics, for which explicit calculations are possible. The way non-renormalizable theories arise in this case is as a low-energy/long-distance approximation in situations for which short- 
distance physics is unimportant, and so is coarse-grained or integrated out [Gell-Mann 1954, Wilson 1974].

For instance, consider the lagrangian density for the quantum electrodynamics of electrons and muons

$$
\mathcal{L}_{Q E D}=-\frac{1}{4} F_{\mu \nu} F^{\mu \nu}-\bar{\psi}\left(\gamma^{\mu} D_{\mu}+m\right) \psi-\bar{\chi}\left(\gamma^{\mu} D_{\mu}+M\right) \chi,
$$

where $m=m_{e}$ and $\psi$ (or $M=m_{\mu} \gg m_{e}$ and $\chi$ ) are the electron (or muon) mass and field. Here $F_{\mu \nu}=\partial_{\mu} A_{\nu}-\partial_{\nu} A_{\mu}$ and $D_{\mu}=\partial_{\mu}+i e A_{\mu}$, as usual, and $\gamma^{\mu}$ represents the Dirac matrices - that satisfy $\left\{\gamma^{\mu}, \gamma^{\nu}\right\}=$ $2 \eta^{\mu \nu}=2 \operatorname{diag}(-,+,+,+)$. This is a renormalizable theory because all parameters, $e, m$ and $M$, have non-positive dimension when regarded as a power of length in fundamental units.

Suppose now we choose to examine observables only involving the electromagnetic interactions of electrons at energies $\omega \ll M$ (such as the energy levels of atoms, for instance). Muons should be largely irrelevant for these kinds of observables, but not completely so. Muons are not completely irrelevant because they can contribute to electron-photon processes at higher orders in perturbation theory as virtual states.

It happens that any such effects due to virtual muons can be described at low energies by the following effective field theory of electrons and photons only:

$$
\begin{aligned}
\mathcal{L}_{\mathrm{eff}} & =-\frac{1}{4} F_{\mu \nu} F^{\mu \nu}-\bar{\psi}\left(\gamma^{\mu} D_{\mu}+m\right) \psi+\frac{k_{1} \alpha}{30 \pi M^{2}} F^{\mu \nu} \square F_{\mu \nu}+\cdots \quad(1.6) \\
& =-\frac{1}{4} F_{\mu \nu} F^{\mu \nu}-\bar{\psi}\left(\gamma^{\mu} D_{\mu}+m\right) \psi+\frac{k_{1} \alpha}{15 \pi M^{2}}\left(\bar{\psi} \gamma_{\mu} \psi\right)\left(\bar{\psi} \gamma^{\mu} \psi\right)+\cdots,
\end{aligned}
$$

where the second line is obtained from the first by performing the field redefinition

$$
A_{\mu} \rightarrow A_{\mu}+\frac{k_{1} \alpha}{15 \pi M^{2}}\left[\square A_{\mu}-i e\left(\bar{\psi} \gamma_{\mu} \psi\right)\right]+\cdots .
$$

In both equations the ellipses describe terms suppressed by more than two powers of $1 / M$.

The lagrangian densities of eqs. (1.5) and (1.6) are precisely equivalent in that they give precisely the same results for all low-energy electron/photon observables, provided one works only to leading order in $1 / M^{2}$. If the accuracy of the agreement is to be at the one-loop level, then equivalence requires the choice $k_{1}=1$, and the effective interaction captures the leading effects of a muon loop in the vacuum polarization. If agreement is to be at the two-loop level, then $k_{1}=1+\mathcal{O}(\alpha)$ captures effects coming from higher loops as well, and so on. 
This example (and many many others) shows that it must be possible to make sensible predictions using non-renormalizable theories. This must be so because the lagrangian of eq. (1.6) is not renormalizable its coupling has dimensions (length) ${ }^{2}$ - yet it agrees precisely with the (very sensible) predictions of QED, eq. (1.5). But it is important that this agreement only works up to order $1 / M^{2}$.

If we work beyond order $1 / M^{2}$ in this expansion, we can still find a lagrangian, $\mathcal{L}_{\text {eff }}$, that captures all of the effects of QED to the desired order. The corresponding lagrangian requires more terms than in eq. (1.6), however, also including terms like

$$
\mathcal{L}_{4}=\frac{k_{2} \alpha^{2}}{90 M^{4}}\left(F_{\mu \nu} F^{\mu \nu}\right)^{2}
$$

that arise at order $1 / M^{4}$. Agreement with QED in this case requires $k_{2}=$ $1+\mathcal{O}(\alpha)$. Sensible predictions can be extracted from non-renormalizable theories, but only if one is careful to work only to a fixed order in the $1 / M$ expansion.

What is useful about this process is that an effective theory like (1.6) is much easier to use than is the full theory (1.5). And any observable whatsoever may be computed once the coefficients $\left(k_{1}\right.$ and $k_{2}$ in the above examples) of the various non-renormalizable interactions are identified. This can be done by comparing its implications with those of the full theory for a few specific observables.

What about the UV divergences associated with these new effective interactions? They must be renormalized, and the many couplings required to perform this renormalization correspond to the many couplings that arise within the effective theory at successive orders in $1 / M$. But predictiveness is not lost because working to fixed order in $1 / M$ means that only a fixed number of effective couplings are required in any given application.

At present this is the only known way to make sense of perturbatively non-renormalizable theories. In particular it means that there is a hidden approximation involved in the use of a non-renormalizable theory - the low-energy, $1 / M$, expansion - that may not have been obvious from the get-go.

\subsubsection{GR as an effective theory}

What would this picture mean if applied to GR? First, it would mean that GR must be regarded as the leading term in the low-energy/long- 
distance approximation to some more fundamental theory. Working beyond leading order would mean extending the Einstein-Hilbert action to include higher powers of curvatures and their derivatives, with the terms with the fewest derivatives being expected to dominate at low energies [for a review see Burgess 2004].

Since we do not know what the underlying theory is, we cannot hope to compute the couplings in this effective theory from first principles as was done above for QED. Instead we treat these couplings as phenomenological, ultimately to be determined from experiment.

The most general interactions involving the fewest curvatures and derivatives, that are consistent with general covariance are

$$
\begin{aligned}
-\frac{\mathcal{L}_{\mathrm{eff}}}{\sqrt{-g}}=\lambda+ & \frac{M_{p}^{2}}{2} R+a_{1} R_{\mu \nu} R^{\mu \nu} \\
+ & a_{2} R^{2}+a_{3} R_{\mu \nu \lambda \rho} R^{\mu \nu \lambda \rho}+a_{4} \square R \\
& \quad+\frac{b_{1}}{m^{2}} R^{3}+\frac{b_{2}}{m^{2}} R R_{\mu \nu} R^{\mu \nu}+\frac{b_{3}}{m^{2}} R_{\mu \nu} R^{\nu \lambda} R_{\lambda}^{\mu}+\cdots
\end{aligned}
$$

where $R^{\mu}{ }_{\nu \lambda \rho}$ is the metric's Riemann tensor, $R_{\mu \nu}=R^{\lambda}{ }_{\mu \lambda \nu}$ is its Ricci tensor, and $R=g^{\mu \nu} R_{\mu \nu}$ is the Ricci scalar, each of which involves precisely two derivatives of the metric.

The first term in eq. (1.9) is the cosmological constant, which we drop because observations imply $\lambda$ is (for some unknown reason, see below) extremely small. Once this is done the leading term in the derivative expansion is the Einstein-Hilbert action whose coefficient, $M_{p}=(8 \pi G)^{-1 / 2}=\left(\sqrt{8 \pi} \ell_{p}\right)^{-1} \sim 10^{18} \mathrm{GeV}$, has dimensions of mass (when $\hbar=c=1$ ), and is set by the value of Newton's constant. This is followed by curvature-squared terms having dimensionless effective couplings, $a_{i}$, and curvature-cubed terms with couplings inversely proportional to a mass, $b_{i} / m^{2}$, (not all of which are written in eq. (1.9)).

Although the numerical value of $M_{p}$ is known, the mass scale $m$ appearing in the curvature-cubed (and higher) terms is not. But since it appears in the denominator it is the lowest mass scale to have been integrated out that should be expected to dominate. What its value should be depends on the scale of the applications one has in mind. For applications to the solar system or to astrophysics $m$ might reasonably be taken to be the electron mass, $m_{e}$. But for applications to inflation, where the scales of interest are much larger than $m_{e}, m$ would instead be taken to be the lightest particle that is heavier than the scales of inflationary interest. 


\subsubsection{Power counting}

The Einstein-Hilbert term should dominate at low energies (since it involves the fewest derivatives), and this expectation can be made more precise by systematically identifying which interactions contribute to a particular order in the semiclassical expansion. To do so we expand the metric about an asymptotically static background spacetime: $g_{\mu \nu}=\bar{g}_{\mu \nu}+2 h_{\mu \nu} / M_{p}$, and compute (say) the scattering amplitudes for asymptotic graviton states that impinge onto the geometry from afar.

If the energy, $\omega$, of the incoming states are all comparable and similar to the curvatures scales of the background spacetime, dimensional analysis can be used to give an estimate for the energy-dependence of an $L$-loop contribution to a scattering amplitude, $\mathcal{A}(\omega)$. Consider a contribution to this amplitude that involves $E$ external lines and $V_{i d}$ vertices involving $d$ derivatives and $i$ attached graviton lines. Dimensional analysis leads to the estimate:

$$
\mathcal{A}(\omega) \sim \omega^{2} M_{p}^{2}\left(\frac{1}{M_{p}}\right)^{E}\left(\frac{\omega}{4 \pi M_{p}}\right)^{2 L} \prod_{i} \prod_{d>2}\left[\frac{\omega^{2}}{M_{p}^{2}}\left(\frac{\omega}{m}\right)^{(d-4)}\right]^{V_{i d}} .
$$

Notice that no negative powers of $\omega$ appear here because general covariance requires derivatives come in pairs, so the index $d$ in the product runs over $d=4+2 k$, with $k=0,1,2, \ldots$.

This last expression displays the low-energy approximation alluded to above because it shows that the small quantities controlling the perturbative expansion are $\omega / M_{p}$ and $\omega / m$. Use of this expansion (and in particular its leading, classical limit - see below) presupposes both of these quantities to be small. Notice also that because $m \ll M_{p}$, factors of $\omega / m$ are much larger than factors of $\omega / M_{p}$, but because they do not arise until curvature-cubed interactions are important, the perturbative expansion always starts off with powers of $\omega / M_{p}$.

\subsubsection{What justifies the classical approximation?}

Eq. (1.10) answers a question that is not asked often enough: What is the theoretical error made when treating gravitational physics in the classical approximation? What makes it so useful in this regard is that it quantifies the size of the contribution to $\mathcal{A}(\omega)$ (or other observables) arising both from quantum effects (i.e. loops, with $L \geq 1$ ), and from terms normally not included in the lagrangian (such as higher-curvature 
terms). This allows an estimate of the size of the error that is made when such terms are not considered (as is often the case).

In particular, eq. (1.10) justifies why classical calculations using GR work so well, and quantifies just how accurate their quantum corrections are expected to be. To see this, we ask which graphs dominate in the small- $\omega$ limit. For any fixed process (i.e. fixed E) eq. (1.10) shows the dominant contributions are those for which

$$
L=0 \quad \text { and } \quad V_{i d}=0 \text { for any } d>2 .
$$

That is, the dominant contribution comes from arbitrary tree graphs constructed purely from the Einstein-Hilbert $(d=2)$ action. This is precisely the prediction of classical General Relativity.

For instance, for the scattering of two gravitons about flat space, $g\left(p_{1}\right)+g\left(p_{2}\right) \rightarrow g\left(p_{1}^{\prime}\right)+g\left(p_{2}^{\prime}\right)$, we have $E=4$, and eq. (1.10) predicts the dominant energy-dependence to be $\mathcal{A}(\omega) \propto\left(\omega / M_{p}\right)^{2}$. This is borne out by explicit tree-level calculations [DeWitt 1967] which give

$$
\mathcal{A}_{\text {tree }}=8 \pi i G\left(\frac{s^{3}}{t u}\right),
$$

for an appropriate choice of graviton polarizations. Here $s=-\left(p_{1}+p_{2}\right)^{2}$, $t=\left(p_{1}-p_{1}^{\prime}\right)^{2}$ and $u=\left(p_{1}-p_{2}^{\prime}\right)^{2}$ are the usual Lorentz-invariant Mandelstam variables built from the initial and final particle four-momenta, all of which are proportional to $\omega^{2}$. This shows both that $\mathcal{A} \sim\left(\omega / M_{p}\right)^{2}$ to leading order, and that it is the physical, invariant, centre-of-mass energy, $\omega_{c m}$, that is the relevant scale against which $m$ and $M_{p}$ should be compared.

The next-to-leading contributions, according to eq. (1.10), arise in one of two ways: either

$$
\begin{aligned}
L & =1 \text { and } V_{i d}=0 \text { for any } d>2 ; \\
\text { or } \quad L & =0, \sum_{i} V_{i 4}=1, \quad \text { and } V_{i d}=0 \text { for } d>4 .
\end{aligned}
$$

These correspond to one-loop (quantum) corrections computed only using Einstein gravity; plus a tree-level contribution including precisely one vertex from one of the curvature-squared interactions (in addition to any number of interactions from the Einstein-Hilbert term). The UV divergences arising in the first type of contribution are absorbed into the coefficients of the interactions appearing in the second type. Both are suppressed compared to the leading, classical, term by a factor of 
$\left(\omega / 4 \pi M_{p}\right)^{2}$. This estimate (plus logarithmic complications due to infrared divergences) is also borne out by explicit one-loop calculations about flat space [Weinberg 1965, Dunbar 1995, Donoghue 1999].

This is the reasoning that shows why it makes sense to compute quantum effects, like Hawking radiation or inflationary fluctuations, within a gravitational context. For observables located a distance $r$ away from a gravitating mass $M$, the leading quantum corrections are predicted to be of order $G \hbar / r^{2} c^{3}=\left(\ell_{p} / r\right)^{2}$. For comparison, the size of classical relativistic corrections is set by $2 G M / r c^{2}=r_{s} / r$, where $r_{s}=2 G M / c^{2}$ denotes the Schwarzschild radius. At the surface of the Sun this makes relativistic corrections of order $G M_{\odot} / R_{\odot} c^{2} \sim 10^{-6}$, while quantum corrections are $G \hbar / R_{\odot}^{2} c^{3} \sim 10^{-88}$. Clearly the classical approximation to GR is extremely good within the solar system.

On the other hand, although relativistic effects cannot be neglected near a black hole, since $2 G M / r_{s} c^{2}=1$, the relative size of quantum corrections near the event horizon is

$$
\left(\frac{\ell_{p}}{r_{s}}\right)^{2}=\frac{G \hbar}{r_{s}^{2} c^{3}}=\frac{\hbar c}{4 G M^{2}}
$$

which is negligible provided $M \gg M_{p}$. Since $M_{p}$ is of order tens of micrograms, this shows why quantum effects represent small perturbations for any astrophysical black holes 1 but would not be under control for any attempt to interpret the gravitational field of an elementary particle (like an electron) as giving rise to a black hole.

\subsubsection{Lessons learned}

What do these considerations tell us about how gravity behaves over very small distances?

The good news is that it says that the observational successes of GR are remarkably robust against the details of whatever small-distance physics ultimately describes gravity over very small distances. This is because any microscopic physics that predicts the same symmetries (like Lorentz invariance) and particle content (a massless spin-2 particle, or equivalently a long-range force coupled to stress-energy) as GR, must be described by a generally covariant effective action like eq. (1.9). Because this is dominated at low energies by the Einstein-Hilbert action,

$\dagger$ Small, but not negligible, since the decrease in mass predicted by Hawking radiation has no classical counterpart with which to compete. 
it suffices to get the low-energy particle content and symmetries right to get GR right in all of its glorious detail [Deser 1970].

The bad news applies to those who think they know what the fundamental theory of quantum gravity really is at small scales, since whatever it is will be very hard to test experimentally. This is because all theories that get the bare minimum right (like a massless graviton), are likely to correctly capture all of the successes of GR in one fell swoop. At low energies the only difference between the predictions of any such theory is the value of the coefficients, $a_{i}$ and $b_{i}$ etc, appearing in the low-energy lagrangian (1.9), none of which are yet observable.

There are two kinds of proposals that allow tests at low energies: those that change the low-energy degrees of freedom (such as by adding new light particles in addition to the graviton - more about these proposals below); and those that change the symmetries predicted for the lowenergy theory. Prominent amongst this latter category are theories that postulate that gravity at short distances breaks Lorentz or rotational invariance, perhaps because spacetime becomes discrete at these scales.

At first sight, breaking Lorentz invariance at short distances seems batty, due to the high accuracy with which experimental tests verify the Lorentz-invariance of the vacuum within which we live. How could the world we see appear so Lorentz invariant if it is really not so deeper down? Surprisingly, experience with other areas of physics suggests this may not be so crazy an idea; we know of other, emergent, symmetries that can appear to be very accurate at long distances even though they are badly broken at short distances. Most notable among these is the symmetry responsible for conservation of baryon number, which has long been known to be an 'accidental' symmetry of the Standard Model. This means that for any microscopic theory whose low-energy particle content is that of the SM, any violations of baryon number must necessarily be described by a non-renormalizable effective interaction [Weinberg 1979a, Wilczek 1979], and so be suppressed by a power of a large inverse mass, $1 / M$. This suppression can be enough to agree with observations (like the absence of proton decay) if $M$ is as large as $10^{16} \mathrm{GeV}$.

Could Lorentz invariance be similarly emergent? If so, it should be possible to find effective field theories for which Lorentz violation first arises suppressed by some power of a heavy scale, $1 / M$, even if Lorentz invariance is not imposed from the outset as a symmetry of the theory. Unfortunately this seems hard to achieve, since in the absence of Lorentz 
invariance it is difficult it in an effective theory to explain why the effective terms

$$
\partial_{t} \psi^{*} \partial_{t} \psi \quad \text { and } \quad \nabla \psi^{*} \cdot \nabla \psi,
$$

should have precisely the same coefficient in the low-energy theory. (See however [Groot Nebbelink 2005] for some attempts.) The problem is that the coefficients of these terms are dimensionless in fundamental units, and so are unsuppressed by powers of $1 / M$. But the relative normalization of these two terms governs the maximal speed of propagation of the corresponding particle, and there are extremely good bounds (for some particles better than a part in $10^{20}$ ) on how much this can differ from the speed of light [see, for instance, Mattingly 2005 for a recent review].

This underlines why proponents of any particular Quantum Gravity proposal must work hard to provide the effective field theory (EFT) that describes their low-energy limit [see Kostelecky 2004, Mattingly 2005 for some gravitational examples]. Since all of the observational implications are contained within the effective theory, it is impossible to know without it whether or not the proposal satisfies all of the existing experimental tests. This is particularly true for proposals that claim to predict a few specific low-energy effects that are potentially observable (such as small violations of Lorentz invariance in cosmology). Even if the predicted effects should be observed, the theory must also be shown not to be in conflict with other relevant observations (such as the absence of Lorentz invariance elsewhere), and this usually requires an EFT formulation.

\subsection{Modifying Gravity over Long Distances}

There also has been considerable activity over recent years investigating the possibility that GR might fail, but over very long distances rather than short ones. This possibility is driven most persuasively from cosmology, where the Hot Big Bang paradigm has survived a host of detailed observational tests, but only if the universe is pervaded by no less than two kinds of new exotic forms of matter: dark matter (at present making up $\sim 25 \%$ of the universal energy density) and Dark Energy (comprising $\sim 70 \%$ of the cosmic energy density). Because all of the evidence for the existence of these comes from their gravitational interactions, inferred

$\dagger$ The situation would be different in Euclidean signature, since then invariance under a lattice group of rotations can suffice to imply invariance under $O(4)$ transformations, at least for the kinetic terms. 
using GR, the suspicion is that it might be more economical to interpret instead the cosmological tests as evidence that GR is failing over long distances.

But since the required modifications occur over long distances, their discussion is performed most efficiently within an effective lagrangian framework. These next paragraphs summarize my personal take on what has been learnt to this point.

\subsubsection{Consistency issues}

An important consideration when trying to modify gravity over long distances is the great difficulty in doing so in a consistent way. Almost all modifications so far proposed run into trouble with stability or unitarity, in that they predict unstable degrees of freedom like 'ghosts,' particles having negative kinetic energy. The presence of ghosts in a low energy theory is generally regarded as poison because it implies there are instabilities. At the quantum level these instabilities usually undermine our understanding of particle physics and the very stability of the vacuum [see Cline 2004 for a calculation showing what can go wrong], but even at the classical level they typically ruin the agreement between the observed orbital decay of binary pulsars and GR predictions for their energy loss into gravitational waves.

The origin of these difficulties seems to be the strong consistency requirements that quantum mechanics and Lorentz invariance impose on theories of massless particles having spin-one or higher [Weinberg 1964, Deser 1970, Weinberg 1980], with static (non-derivative) interactions. A variety of studies indicate that a consistent description of particles with spins $\geq 1$ always requires a local invariance, which in the cases of spins $1,3 / 2$ and 2 corresponds to gauge invariance, supersymmetry or general covariance, and this local symmetry strongly limits the kinds of interactions that are possible Although it remains an area of active research [Dvali 2000], at present the only systems known to satisfy these consistency constraints consist of relativistic theories of spins 0 through 1 coupled either to gravity or supergravity (possibly in more than 4 spacetime dimensions).

$\dagger$ The AdS/CFT correspondence [Maldacena 1998] - a remarkable equivalence between asymptotically anti-de Sitter gravitational theories and non-gravitational systems in one lower dimensions - may provide a loophole to some of these arguments, although its ultimate impact is not yet known. 


\subsubsection{Dark Matter}

As might be expected, widespread acceptance of the existence of a hitherto-unknown form of matter requires the concordance of several independent lines of evidence, and this constrains one's options when formulating a theory for dark matter. It is useful to review this evidence when deciding whether it indicates a failure of GR or a new form of matter.

The evidence for dark matter comes from measuring the amount of matter in a region as indicated by how things gravitate towards it, and comparing the result with the amount of matter that is directly visible. Several types of independent comparisons consistently point to there being more than 10 times as much dark, gravitating material in space than is visible

- Galaxies: The total mass in a galaxy may be inferred from the orbital motion of stars and gas measured as a function of distance from the galactic center. The results, for large galaxies like the Milky Way, point to several times more matter than is directly visible.

- Galaxy Clusters: Similar measurements using the motion of galaxies and temperature of hot gas in large galaxy clusters also indicate the presence of much more mass than is visible.

- Structure Formation: Present-day galaxies and galaxy clusters formed through the gravitational amplification of initially-small primordial density fluctuations. In this case the evidence for dark matter arises from the interplay of two facts: First, the initial density fluctuations are known to be very small, $\delta \rho / \rho \sim 10^{-5}$, at the time when the CMB was emitted. Second, small initial fluctuations cannot be amplified by gravity until the epoch where non-relativistic matter begins to dominate the total energy density. But this does not give enough time for the initially-small fluctuations to form galaxies unless there is much more matter present than can be accounted for by baryons. The amount required agrees with the amount inferred from the previous measures described above.

These in themselves do not show that the required dark matter need be exotic, the evidence for which also comes from several sources

- Primordial Nucleosynthesis: The total mass density of ordinary mat-

$\dagger$ This is consistent with the cosmological evidence that dark matter is roughly 5 times more abundant than ordinary matter (baryons) because most of the ordinary matter is also dark, and so is also not visible. 
ter (baryons) in the universe can be inferred from the predicted relative abundance of primordial nuclei created within the Hot Big Bang. This predicted abundance agrees well with observations, and relies on the competition between nuclear reaction rates and the rate with which the universe cools. But both of these rates themselves depend on the net abundance of baryons in the universe: the nuclear reaction rates depend on the number of baryons present; and the cooling rate depends on how fast the universe expands, and so - at least, in GR - on its total energy density. The success of the predictions of Big Bang Nucleosynthesis (BBN) therefore fixes the fraction of the universal energy density which can consist of baryons, and implies that there can at most be a few times more baryons than what would be inferred by counting those that are directly visible.

- The Cosmic Microwave Background (CMB): CMB photons provide an independent measure of the total baryon abundance. They do so because sound waves in the baryon density that are present when these photons were radiated are observable as small temperature fluctuations. Since the sound-wave properties depend on the density of baryons, a detailed understanding of the CMB temperature spectrum allows the total baryon density to be reconstructed. The result agrees with the BBN measure described above.

There are two main options for explaining these observations. Since dark matter is inferred gravitationally, perhaps the laws of gravity differ on extra-galactic scales than in the solar system. Alternatively, there could exists a cosmic abundance of a new type of hitherto-undiscovered particle.

At present there are several reasons that make it more likely that dark matter is explained by the presence of a new type of particle than by changing GR on long distances. First, as mentioned above, sensible modifications are difficult to make at long distances that lack ghosts and other inconsistencies. Second, no phenomenological modification of gravity has yet been proposed that accounts for all the independent lines of evidence given above (although there is a proposal that can explain the rotation of galaxies [Milgrom 1983, Sanders 2002]).

On the other hand, all that is required to obtain dark matter as a new form of matter is the existence of a new type of stable elementary particle having a mass and couplings similar to those of the $Z$ boson, which is already known to exist. $Z$ bosons would be excellent dark matter candidates if only they did not decay. A particle with mass 
and couplings like the $Z$ boson, but which is stable - called a Weakly Interacting Massive Particle (WIMP) — would naturally have a relic thermal abundance in the Hot Big Bang that lies in the range observed for dark matter [for a review, see Eidelman 2004]. New particles with these properties are actually predicted by many current proposals for the new physics that is likely to replace the Standard Model at energies to be explored by the Large Hadron Collider (LHC).

At the present juncture the preponderance of evidence - the simplicity of the particle option and the difficulty of making a modification to GR that works - favours the interpretation of cosmological evidence as pointing to the existence of a new type of matter rather than a modification to the laws of gravity.

\subsubsection{Dark Energy}

The evidence for dark energy is more recent, and incomplete, than that for dark matter. At present the evidence for its existence comes from two independent lines of argument:

- Universal Acceleration: Since gravity is attractive, one expects an expanding universe containing only ordinary (and dark) matter and radiation to have a decelerating expansion rate. Evidence for dark energy comes from measurements indicating the universal expansion is accelerating rather than decelerating, obtained by measuring the brightness of distant supernovae [Perlmutter 1997, Riess 1997, Bahcall 1999]. According to GR, accelerated expansion implies the universe is dominated by something with an equation of state satisfying $p<$ $-\rho / 3$, which is not true for ordinary matter, radiation or dark matter.

- Flatness of the universe: An independent measure of the dark energy comes from the observed temperature fluctuations in the CMB. Because the $\mathrm{CMB}$ photons traverse the entire observable universe before reaching us, their properties on arrival depend on the geometry of the universe as a whole (and so also, according to GR, on its total energy density). Agreement with observations implies the total energy density is larger than the ordinary and dark matter abundances, which fall short by an amount consistent with the amount of dark energy required by the acceleration of the universe's expansion [Komatsu 2009].

Again the theoretical options are the existence of a new form of energy density, or a modification of GR at long distances. Although there are 
phenomenological proposals for modifications that can cause the universe to accelerate (such as [Dvali 2000]), all of the previously described problems with long-distance modifications to GR also apply here.

By contrast, there is a very simple energy density that does the job, consisting simply of a cosmological constant - i.e. a constant $\lambda \simeq$ $\left(3 \times 10^{-3} \mathrm{eV}\right)^{4}$ in eq. (1.9), for which $p=-\rho$. This is phenomenologically just what the doctor ordered, and agrees very well with the observations.

The theoretical difficulty here is that a cosmological constant is indistinguishable from the energy density of a Lorentz-invariant vacuum, since both contribute to the stress tensor an amount $T_{\mu \nu}=\lambda g_{\mu \nu}$. In principle, this should be a good thing because we believe we can compute the vacuum energy. The problem is that ordinary particles (like the electron) contribute such an enormous amount - the electron gives $\delta \lambda \simeq m_{e}^{4} \simeq\left(10^{6} \mathrm{eV}\right)^{4}$ - that agreement with the observed value requires a cancellation [Weinberg 1989] to better than one part in $10^{36}$.

\subsubsection{Lessons learned}

Dark matter and dark energy are two forms of exotic matter, whose existence is inferred purely from their gravitational influence on visible objects. It is tempting to replace the need for two new things with a single modification to gravity over very large distances.

Yet the preponderance of evidence again argues against this point of view. First, it is difficult to modify GR at long distances without introducing pathologies. Second, it is difficult to find modifications that account for more than one of the several independent lines of evidence (particularly for dark matter). By contrast, it is not difficult to make models of dark matter (WIMPs) or dark energy (a cosmological constant). For dark energy this point of view runs up against the cosmological constant problem, which might indicate the presence of observably large extra dimensions, but for which no consensus yet exists.

$\dagger$ The only known loophole to this arises if extra dimensions exist, and are as large as 10 microns in size, because in this case the vacuum energy can be localized in the extra dimensions, and so curve these rather than the dimensions we see [Arkani-Hamed 2000, Kachru 2000, Carroll 2003, Aghababaie 2004]. Whether this, together with supersymmetry, can solve the problem is under active study [Burgess 2005]. 


\subsection{Conclusions}

In summary, modifications to General Relativity are widely mooted over both large and small distances. This chapter argues that modifications at small distances are indeed very likely, and well worth seeking. But unless the modification takes place just beyond our present experimental reach $\left(\sim 10^{-19} \mathrm{~m}\right)$ [Arkani-Hamed 1998, Antoniadis 1998, Burgess 2005], it is also likely to be very difficult to test experimentally. The basic obstruction is the decoupling from long distances of short-distance physics, a property most efficiently expressed using effective field theory methods. The good news is that this means that the many observational successes of GR are insensitive to the details of whatever the modification proves to be.

Modifications to GR over very long distances are also possible, and have been argued as more economical than requiring the existence of two types of unknown forms of matter (dark matter and dark energy). If so, consistency constraints seem to restrict the possibilities to supplementing GR by other very light spin-0 or spin-1 bosons (possibly in higher dimensions). The experimental implications of such modifications are themselves best explored using effective field theories. Unfortunately, no such a modification has yet been found that accounts for all of the evidence for dark matter or energy in a way that is both consistent with other tests of GR and is more economical than the proposals for dark matter or energy themselves.

To the extent that the utility of effective field theory relies on decoupling, one might ask: What evidence do we have that Planck-scale physics decouples? There are two lines of argument that bear on this question. First, once specific modifications to gravity are proposed it becomes possible to test whether decoupling takes place. Perhaps the best example of a consistent modification to gravity at short distances is string theory, and all the present evidence points to decoupling holding in this case. But more generally, if sub-Planckian scales do not decouple, one must ask: Why has science made progress at all? After all, although Nature comes to us with many scales, decoupling is what ensures we don't need to understand them all at once. If sub-Planckian physics does not decouple, what keeps it from appearing everywhere, and destroying our hard-won understanding of Nature? 


\section{Acknowledgements}

I thank the editors for their kind invitation to contribute to this volume, and for their patience in awaiting my contribution. My understanding of this topic was learned from Steven Weinberg, who pioneered effective field theory techniques, and was among the first to connect the dots explicitly about gravity's interpretation as an effective field theory. My research is funded by the Natural Sciences and Engineering Research Council of Canada, as well as by funds from McMaster University and Perimeter Institute.

\section{References}

Aghababaie, Y., Burgess, C.P., Parameswaran, S.L. and Quevedo, F. (2004) Nucl. Phys. B 680, 389 arXiv:hep-th/0304256.

Antoniadis, I., Arkani-Hamed, N., Dimopoulos, S. and Dvali, G. (1998) Phys. Lett. B 436257 arXiv:hep-ph/9804398.

Arkani-Hamed, N., Dimopoulos, S. and Dvali, G. (1998) Phys. Lett. B 429 263 arXiv:hep-ph/9803315.

Arkani-Hamed, N., Dimopoulos, S., Kaloper, N. and Sundrum, R. (2000) Phys. Lett. B 480 193, hep-th/0001197.

Bahcall, N., Ostriker, J.P., Perlmutter, S. and Steinhardt, P.J. (1999) Science 284 1481, astro-ph/9906463.

Burgess, C.P. (2004) Living Rev. Rel. 75 gr-qc/0311082.

Burgess, C.P. (2005) AIP Conf. Proc. 743, 417 arXiv:hep-th/0411140.

Carroll, S.M. and M. M. Guica, M.M. , arXiv:hep-th/0302067.

Cline, J.M., Jeon, S. and Moore, G.D. (2004) Phys. Rev. D 70043543 arXiv:hep-ph/0311312.

Deser, S. (1970) Gen. Rel. Grav. 19 arXiv:gr-qc/0411023.

DeWitt, B.S. (1967) Phys. Rev. 1621239.

Donoghue, J.F. and Torma, T. (1999) Phys. Rev. 060024003 hep-th/9901156.

Dunbar, D.C. and Norridge, P.S. (1995) Nucl. Phys. B433 181.

Dvali, G., Gabadadze, G. and Porrati, M. (2000) Phys. Lett. B485 208-214 hep-th/0005016.

Eidelman, S. et al. (2004) Review of Particle Properties, Phys. Lett. B592 1.

Gasser, G. and Leutwyler, H. (1984) Annals of Physics (NY) 158142.

Gell-Mann, M. and Low, F.E. (1954) Phys. Rev. 951300.

Groot Nibbelink, S. and Pospelov, M. (2005) Phys. Rev. Lett. 94081601 arXiv:hep-ph/0404271.

Kachru, S., Schulz, M.B. and Silverstein, E. (2000) Phys. Rev. D 62045021 , hep-th/0001206.

Komatsu, E. et al. (2009), ApJS, 180, 330-376, arXiv:0803.0547.

Kostelecky, V. A. (2004) Phys. Rev. D 69105009 arXiv:hep-th/0312310.

Maldacena, J.M. (1998) Adv. Theor. Math. Phys. 2 (1998) 231 [nt. J. Theor. Phys. 38 (1999) 1113] arXiv:hep-th/9711200.

Mattingly, D. (2005) Living Rev. Rel. 85 arXiv:gr-qc/0502097.

Milgrom, M. (1983) Ap. J. 270 365-370; 371-283; 384-389.

Perlmutter, S., et al. (1997) Ap. J. 483565 astro-ph/9712212. 
Riess, A.G., et al (1997) Ast. J. 1161009 astro-ph/9805201.

Sanders, R.H. and McGaugh, S.S (2002) Ann. Rev. Astron. Astrophys. 40 263-317, astro-ph/0204521.

Weinberg, S. (1964) Phys. Rev. 134 B882.

Weinberg, S. (1965) Phys. Rev. 140516.

Weinberg, S. (1979) Physica 96A 327.

Weinberg, S. (1979a) Phys. Rev. Lett. 431566.

Weinberg, S. and Witten, E. (1980) Phys. Lett. B 9659.

Weinberg, S. (1989) Rev. Mod. Phys. 611.

Wilczek, F. and Zee, A. (1979) Phys. Rev. Lett. 431571.

Will, C.M. (2001), Living Rev. Rel. 44 gr-qc/0103036].

Wilson, K.G. and Kogut, J.B. (1974) Phys. Rept. 1275 